\title{
Characteristics of esophageal cancer in patients with head and neck squamous cell carcinoma
}

\author{
Lei Wang ${ }^{1}$, Wenjing Pang ${ }^{1}$, Kun Zhou $^{1}$, Lei Li ${ }^{1}$, Feng Wang ${ }^{2}$, Wei Cao ${ }^{3}$, Xiangjun Meng ${ }^{1}$ \\ ${ }^{1}$ Department of Gastroenterology, Shanghai Ninth Peoples' Hospital, Shanghai Jiao Tong University, Shanghai, China; ${ }^{2}$ Department of Thoracic \\ Surgery, Shanghai Ninth Peoples' Hospital, Shanghai Jiao Tong University, Shanghai, China; ${ }^{3}$ Department of Oral and Maxillofacial-Head and Neck \\ Oncology, Shanghai Ninth Peoples' Hospital, Shanghai Jiao Tong University, Shanghai, China \\ Contributions: (I) Conception and design: L Wang, X Meng; (II) Administrative support: None; (III) Provision of study materials or patients: F Wang, \\ W Cao; (IV) Collection and assembly of data: W Pang, K Zhou; (V) Data analysis and interpretation: L Li; (VI) Manuscript writing: All authors; (VII) \\ Final approval of manuscript: All authors. \\ Correspondence to: Xiangjun Meng. Department of Gastroenterology, Shanghai Ninth Peoples' Hospital, Shanghai Jiao Tong University, 639 Zhizaoju \\ Road, Shanghai 200011, China. Email: meng_xiangjun@yahoo.com; Wei Cao. Department of Oral and Maxillofacial-Head and Neck Oncology, \\ Shanghai Ninth Peoples' Hospital, Shanghai Jiao Tong University, 639 Zhizaoju Road, Shanghai 200011, China. Email: caowei561521@hotmail.com.
}

Background: We investigated the clinicopathological features of esophageal cancer in patients with a history of head and neck squamous cell carcinoma (HNSCC) with the intention of providing information regarding the characteristics of these patients.

Methods: A retrospective study was performed in 32 cases of esophageal cancer with HNSCC who were diagnosed using upper gastrointestinal endoscopy between 2007 to 2017. Synchronous carcinoma (SC) group and metachronous carcinoma (MC) group was established based on whether esophageal cancer was diagnosed within 6 months after HNSCC diagnosis. The clinicopathological features of esophageal cancer and HNSCC, as well as follow-up treatment and survival, were analyzed in esophageal cancer patients in both groups.

Results: There were 8 cases of 8 patients ( 7 males and 1 female) in the SC group and 24 cases of 22 patients (21 males and 1 female) in the MC group. The majority of esophageal cancer of HNSCC were male patients aged 50-69 years. The average interval time between diagnosis of esophageal cancer and HNSCC was $36.0 \pm 39.2$ months $(3.25 \pm 2.19$ months for the SC group and $46.90 \pm 39.73$ months for the MC group). Ninety-three-point-seven-five percent (30/32) of the patients had esophageal cancer within 6 years after HNSCC. The proportion of early esophageal cancer and successful surgical treatment in the SC group was significantly higher compared to the MC group $(\mathrm{P}<0.05)$.

Conclusions: Detection of esophageal cancer should be prioritized in HNSCC patients.

Keywords: Retrospective study; esophageal neoplasms; mouth neoplasms; endoscopy

Submitted Sep 13, 2020. Accepted for publication Apr 08, 2021.

doi: $10.21037 /$ tcr-20-2880

View this article at: http://dx.doi.org/10.21037/tcr-20-2880

\section{Introduction}

Patients with head and neck squamous cell carcinoma (HNSCC) are known to have a high prevalence of a second primary carcinoma in their upper gastrointestinal tract, most commonly in the esophagus $(1,2)$. The second primary carcinoma can be divided into synchronous and metachronous tumors. Synchronous carcinoma (SC) is defined as the occurrence of a second primary cancer within the first 6 months following the detection of the first cancer, whereas metachronous carcinoma (MC) appear after 6 months (3). The development of esophageal squamous cell carcinoma (ESCC) as reported in several previous studies manifests in about $5-15 \%$ of HNSCC patients, 
which is higher compared to the general population (4-8). ESCC of these patients often leads to treatment failure of HNSCC resulting in a poor prognosis due to the absence of esophageal malignant tumor-related clinical symptoms and early detection (9-11).

Early detection of esophageal cancer is possible with advances in endoscopy such as NBI in combination with magnifying endoscopy, and routine esophageal screening in asymptomatic patients of HNSCC has been recommended in several previous reports (12-18), however, it is unclear how long and for which patients screening for esophageal cancer is necessary $(19,20)$. Hence, we conducted a retrospective investigation of ESCC in HNSCC patients to further investigate the characteristics of the disease and provide a basis for effective clinical screening strategies. We present the following article in accordance with the STROBE reporting checklist (available at http://dx.doi. org/10.21037/tcr-20-2880).

\section{Methods}

\section{Study design}

We retrospectively reviewed the medical records of 32 patients with esophageal cancer with a history of HNSCC diagnosed at the Department of Gastroenterology, Shanghai Ninth Peoples' Hospital, Shanghai Jiao Tong University (Shanghai, China) from 2007 to 2017. All esophageal cancers were diagnosed after patients underwent upper gastrointestinal endoscopy due to progressive dysphagia or pain behind the sternum. Inclusion criteria were as follows: (I) esophageal lesions were observed by upper gastrointestinal endoscopy with NBI mode; (II) histopathology of esophageal lesions was confirmed as malignant or high-grade intraepithelial neoplasia (HGIN); (III) retrospective review of patients with HNSCC history were diagnosed by histopathology. Exclusion criteria were as follows: patients who had a history of esophageal cancer before HNSCC diagnosis. Based on the interval time from HNSCC diagnosis to esophageal cancer diagnosis, synchronous esophageal cancers were defined as occurring within 6 months following HNSCC diagnosis and metachronous esophageal cancers occurring after 6 months. The study was conducted in accordance with the Declaration of Helsinki (as revised in 2013). The study was approved by the ethics committee of Shanghai Ninth People's Hospital, Shanghai Jiao Tong University School of Medicine (No.: SH9H-2020-T189-1), and individual consent for this retrospective analysis was waived.

\section{Treatment and staging of HNSCC patients}

All HNSCC patients underwent surgical treatment at the Department of Oral and Maxillofacial-Head and Neck Oncology, Shanghai Ninth Peoples' Hospital, Shanghai Jiao Tong University (Shanghai, China). Histopathological diagnoses were made at the Department of Oral Pathology of our hospital. HNSCC was classified according to the histopathological results of surgery and the TNM staging system for lip and oral cancer (AJCC eighth edition) (21). Radiotherapy history for each HNSCC patient was also reviewed.

\section{Endoscopic examination and classification of esophageal cancer}

Upper gastrointestinal endoscopy (GIF-H290; Olympus, Japan) was performed through the mouth by a doctor proficient in endoscopy. Once in the oral cavity, the NBI mode was turned on to observe the pyriform sinus and esophageal mucosa and focused on suspected lesions in the esophagus. A biopsy was performed when deemed necessary for a definitive pathological diagnosis. The classification of esophageal cancer was based on the AJCC eighth edition standard (21). Gastrointestinal endoscopy was performed at initial diagnosis of HNSCC, or on postoperative follow-up.

\section{Patient follow-up}

All patients who were diagnosed with esophageal cancer by upper gastrointestinal endoscopy were followed up by trained medical personnel by telephone for follow-up treatment and prognosis for at least 36 months or until patient death.

\section{Statistical analysis}

Comparisons between the SC and MC groups were performed using the $\chi^{2}$ test or one-way ANOVA. The statistical analysis was performed using GraphPad Prism 6 and $\mathrm{P}$ values less than 0.05 were considered significant. Survival analyses of the SC and MC group were performed using GraphPad Prism 6, and survival time was calculated from the time of esophageal cancer diagnosis. The endpoint was 36 months after an esophageal cancer diagnosis or patient death. 
Table 1 Characteristics of the 30 HNSCC patients (mean \pm SD)

\begin{tabular}{|c|c|c|c|c|}
\hline Characteristics & Total $(n=30)$ & $\begin{array}{l}\text { Synchronous carcinoma } \\
\text { group, } \mathrm{SC}(\mathrm{n}=8)\end{array}$ & $\begin{array}{l}\text { Metachronous carcinoma } \\
\text { group, MC }(n=22)\end{array}$ & $\mathrm{P}$ \\
\hline Sex & & & & 0.4399 \\
\hline Female & 2 & 1 & 1 & \\
\hline Primary cancer location & & & & 0.0167 \\
\hline Tongue & 10 & 1 & 9 & \\
\hline Mouth floor & 6 & 1 & 5 & \\
\hline Larynx & 1 & 0 & 1 & \\
\hline HNSCC stage & & & & 0.3740 \\
\hline Stage I & 10 & 2 & 8 & \\
\hline Stage II & 7 & 1 & 6 & \\
\hline Stage III & 8 & 4 & 4 & \\
\hline Stage IV & 5 & 1 & 4 & \\
\hline Radiotherapy & & & & 0.5439 \\
\hline
\end{tabular}

HNSCC, head and neck squamous cell carcinoma; SD, standard deviation; SC, synchronous carcinoma; MC, metachronous carcinoma.

\section{Results}

\section{Characteristics of patients included in the retrospective study}

Thirty-two esophageal cancers were present in 30 HNSCC patients, and cases of esophageal cancer occurred repeatedly in 2 HNSCC patients. The gender, age of HNSCC patients, and esophageal cancer cases are summarized in Tables 1,2. There were no significant differences in gender and age between the SC and MC groups.

\section{Clinicopathological features of HNSCC}

With regards to HNSCC stage, for the SC group, there were 2 cases with stage I, 1 case with stage II, 4 cases with stage III, and 1 case with stage IV A; for the MC group, there were 8 cases with stage I, 6 cases with stage II, 4 cases with stage III, and 4 cases with stage IVA. There were no significant differences in HNSCC staging between the SC and MC groups (Table 1). Three HNSCC patients underwent postoperative radiotherapy in the SC group, and 11 HNSCC patients underwent postoperative radiotherapy in the MC group. There were no significant differences in radiotherapy history between the $\mathrm{SC}$ and $\mathrm{MC}$ groups (Table 1).

The location distribution of HNSCC in the SC group was as follows: 1 case on the tongue, 1 case on the mouth floor, 5 cases on the soft palate, and 1 case on the lip. The location distribution of HNSCC in the MC group was as follows: 9 cases on the tongue, 5 cases on the mouth floor, 5 cases on the gingiva, 2 cases on the soft palate, and 1 case on the larynx. The most common HNSCC tumor location 
Table 2 Characteristics of the 32 esophageal cancer cases with HNSCC history (mean \pm SD)

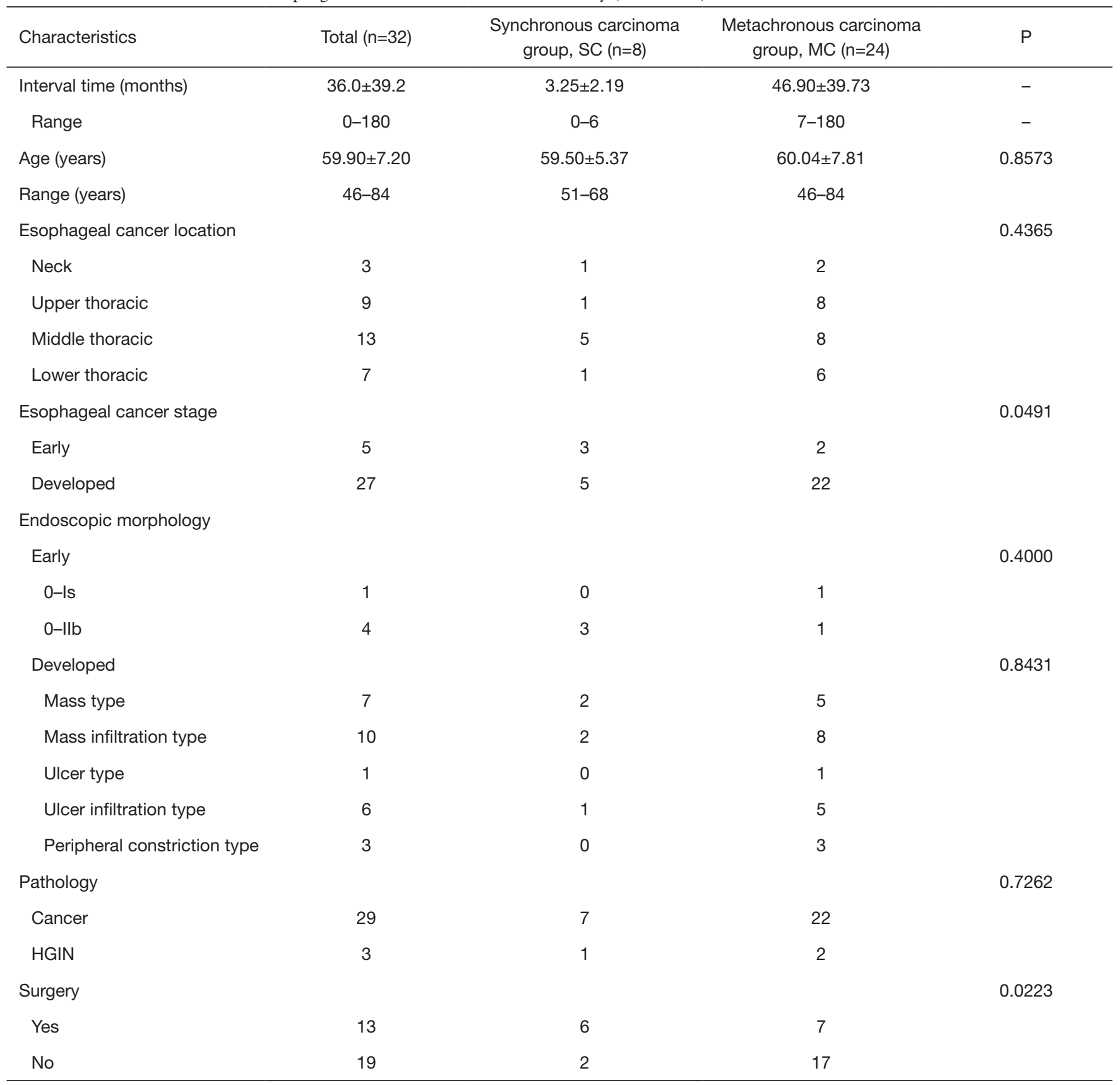

HNSCC, head and neck squamous cell carcinoma; SD, standard deviation; SC, synchronous carcinoma; MC, metachronous carcinoma; HGIN, high-grade intraepithelial neoplasia.

in the SC group was on the soft palate, while the most common HNSCC tumor location in the MC group was on the tongue. There were significant differences in the location distribution of HNSCC between the SC and MC groups $(\mathrm{P}<0.05)($ Table 1, Figure $1 A)$.

\section{Clinicopathological features of esophageal cancer}

The average interval time between diagnosis of esophageal cancer and HNSCC was $36.0 \pm 39.2$ months $(3.25 \pm 2.19$ months for the SC group and $46.90 \pm 39.73$ months for the MC group). The longest interval time was 180 months (Table 2). 

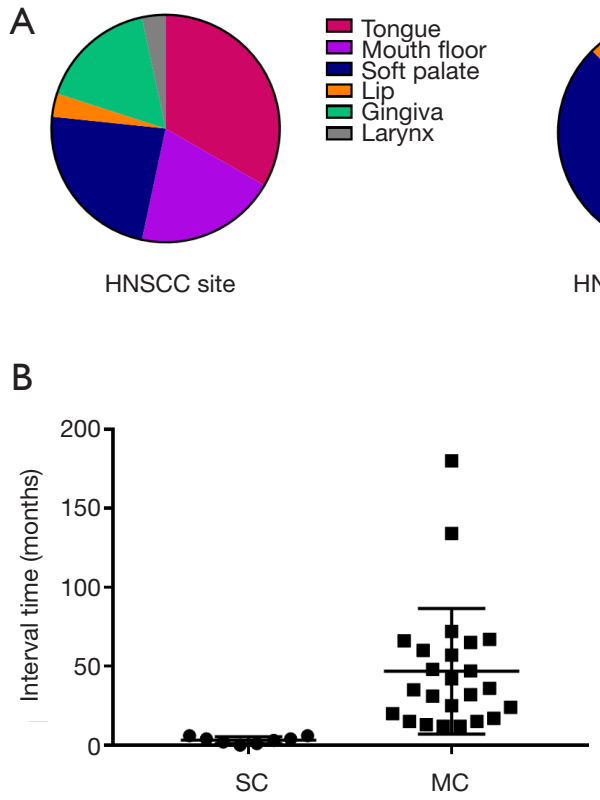
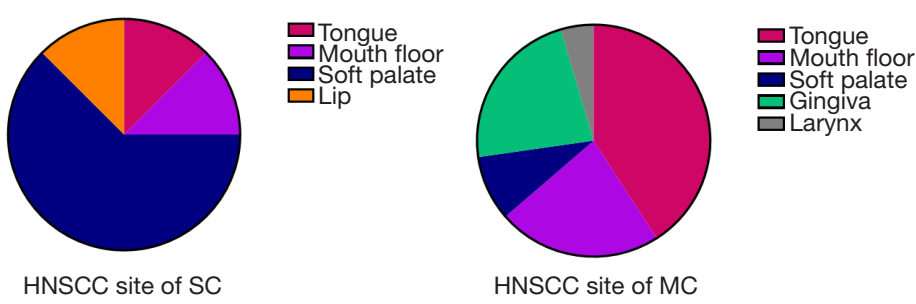

C

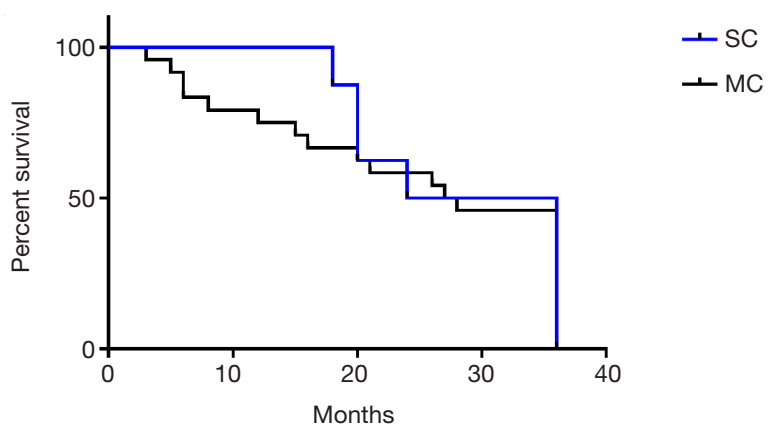

Figure 1 Characteristics and differences between the SC and MC esophageal cancer patients with HNSCC. (A) Pie charts represent the significant differences in the location of HNSCC between the SC and MC group. (B) Scatter plots showing that 93.75\% [30/32] of esophageal cancers occurred within 6 years of HNSCC diagnosis. (C) Survival analysis demonstrating no significant differences in survival time between the MC and SC group. HNSCC, head and neck squamous cell carcinoma; SD, standard deviation; SC, synchronous carcinoma; MC, metachronous carcinoma.

For the 8 cases in the SC group, there was 1 case with an interval time of 0 month, 1 case with 1 month, 1 case with 2 months, 1 case with 3 months, 2 cases with 4 months, and 2 cases with 6 months. For the 24 cases in the MC group, the interval times were $12,12,13,15,15,17,20,24,25$, $31,32,35,36,42,47,48,57,60,65,66,67,72,134$, and 180 months. For the 2 cases with interval times over 72 months, 1 case had a second occurrence of esophageal cancer. Overall, 93.75\% [30/32] of esophageal cancers occurred within 6 years (72 months) after HNSCC diagnosis (Table 2, Figure 1B).

Regarding the location of the esophageal cancers, for the SC group, there was 1 case of cancer located in the cervical esophagus, 1 case in the upper thoracic esophagus, 5 cases in the middle thoracic esophagus, and 1 case in the lower thoracic esophagus. For the MC group, there were 2 cases of cancer located in the cervical esophagus, 8 cases in the upper thoracic esophagus, 8 cases in the middle thoracic esophagus, and 6 cases in the lower thoracic esophagus. There were no significant differences between the two groups in terms of the location of esophageal cancer. The most common location was in the middle thoracic segment of the esophagus (Table 2).

In terms of esophageal cancer staging, there were 3 cases with early esophageal cancer and 5 cases with advanced esophageal cancer in the SC group. There were 2 cases of early esophageal cancer and 22 cases of advanced esophageal cancer in the MC group. The proportion of early esophageal cancer in the SC group $(37.5 \%, 3 / 8)$ was significantly higher compared to the MC group (8.3\%, 2/24) $(\mathrm{P}<0.05)($ Table 2).

In terms of endoscopic morphology, three cases of early esophageal cancer in the SC group were of the 0-IIb type, and 2 cases of early esophageal cancer in the MC group were $0-$ IIb type and $0-$ Is type. The 5 cases of advanced esophageal cancer in the SC group were mass type (2 cases), mass infiltration type ( 2 cases), and ulcer infiltration type ( 1 case). The 22 cases of advanced esophageal cancer in the MC group were mass type (5 cases), mass infiltration type ( 8 cases), ulcer type ( 1 case), ulcer infiltration type (5 cases), and constriction type (3 cases). The endoscopic morphology of esophageal cancer was mainly mass type and mass infiltration type. There were no significant differences between the two groups (Table 2). 
In terms of histopathological findings of the esophageal lesions, there were 7 cases of squamous cell carcinoma and 1 case of HGIN in the SC group. There were 22 cases of squamous cell carcinoma and 2 cases of HGIN in the MC group (Table 2).

\section{Follow-up treatment and survival analysis of patients with esophageal cancer}

For the SC group, 4 cases underwent surgery, 1 case underwent surgery + chemotherapy + radiotherapy, 1 case underwent surgery + radiotherapy, 1 case underwent radiotherapy, and 1 case only received percutaneous endoscopic gastrostomy (PEG). For the MC group, 2 cases underwent surgery (surgery to remove the tumor was unsuccessful for one case), 2 cases underwent surgery + chemotherapy + radiotherapy, 4 cases underwent surgery + chemotherapy, 1 case underwent chemotherapy, 1 case received PEG, 1 case received targeted therapy, and remaining cases received palliative care. The proportion of surgeries for esophageal cancer in the SC group (75\%, 6/8) was significantly higher compared to the MC group (29.2\%, $7 / 24)(\mathrm{P}<0.05)$ (Table 2). Survival analysis demonstrated no significant differences in survival time between the MC and SC groups (Figure1C).

\section{Discussion}

In recent years, the field of cancerization of tumors has captured the attention of researchers (22-24). Esophageal and oral mucosa epithelial cells are both squamous epithelial cells and are exposed to similar environments. Hence, esophageal cancers are closely related to HNSCC (25). To determine the clinicopathological features of esophageal cancer in patients with HNSCC, we retrospectively analyzed esophageal cancer cases with a history of HNSCC who were diagnosed using upper gastrointestinal endoscopy in our hospital from 2007 to 2017.

Because the overall number of HNSCC cases was difficult to determine, we were unable to determine the specific incidence of esophageal cancer in HNSCC patients. However, from previous studies, the incidence of esophageal cancer in HNSCC patients was observed to be $5-15 \%$. This is significantly higher compared to the general population (4-8). With regards to the 32 cases of recurrent esophageal cancer in our present study, 29 cases were between the ages of 50-69, accounting for $90.6 \%$ of the cases. Furthermore, male cases were significantly higher compared to female cases and were consistent with previously published reports (26). This may be due to males being more likely to smoke and drink compared to females (27-29).

Concerning HNSCC characteristics of patients developing esophageal secondary primary cancers, a previous study demonstrated that esophageal cancers were not associated with HNSCC location (30). However, another study demonstrated that a larger number of esophageal secondary primary cancers were detected in patients with hypopharyngeal and oropharyngeal cancers (1). Our retrospective study showed that HNSCC cases with concurrent esophageal cancers were most common in the soft palate, and HNSCC with metachronous esophageal cancers were mostly found in the tongue. The reasons for these localizations remain to be deciphered. Furthermore, our study demonstrated no significant relationship between recurrent time of esophageal cancers and HNSCC staging. However, some previous studies showed that patients with esophageal recurrent cancers had earlier HNSCC staging. This may be because HNSCC patients with earlier stages had adequate survival time to develop esophageal recurrent cancers (31).

In addition, we showed that the manifestation period of esophageal cancers was large and ranged from concurrence with HNSCC to 15 years after HNSCC. However, $93.75 \%$ [30/32] of esophageal cancers occurred within 6 years of HNSCC diagnosis. Regarding preoperative examination of HNSCC, CT examinations to exclude second primary cancers of the lungs have been widely used $(32,33)$. However, it is still grossly inadequate for the exclusion of concurrent esophageal cancer compared to upper gastrointestinal endoscopy. Due to the long timespan of metachronous esophageal cancers, the vigilance and compliance of doctors and patients will decrease, making it more difficult for the early diagnosis of metachronous esophageal cancers. In this study, the proportion of earlystage cancers in the MC group were significantly lower compared to the SC group. Furthermore, the proportion of surgical treatment in the MC group was significantly lower compared to the SC group. Hence it is critical for patients to have regular follow-up management after HNSCC surgery. Targeted upper gastrointestinal endoscopy is expected to improve the early diagnosis rate and reduce mortality in patients with metachronous esophageal cancers.

One of the limitations of this retrospective study was the very limited sample size. Larger multicenter prospective studies should be performed to validate our findings. This will be critical for detecting esophageal cancers in HNSCC 
patients more effectively.

\section{Acknowledgments}

Funding: None.

\section{Footnote}

Reporting Checklist: The authors have completed the STROBE reporting checklist. Available at http://dx.doi. org/10.21037/tcr-20-2880

Data Sharing Statement: Available at http://dx.doi. org/10.21037/tcr-20-2880

Peer Review File: Available at http://dx.doi.org/10.21037/tcr20-2880

Conflicts of Interest: All authors have completed the ICMJE uniform disclosure form (available at http://dx.doi. org/10.21037/tcr-20-2880). The authors have no conflicts of interest to declare.

Ethical Statement: The authors are accountable for all aspects of the work in ensuring that questions related to the accuracy or integrity of any part of the work are appropriately investigated and resolved. The study was conducted in accordance with the Declaration of Helsinki (as revised in 2013). The study was approved by the ethics committee of Shanghai Ninth People's Hospital, Shanghai Jiao Tong University School of Medicine (No.: SH9H2020-T189-1), and individual consent for this retrospective analysis was waived.

Open Access Statement: This is an Open Access article distributed in accordance with the Creative Commons Attribution-NonCommercial-NoDerivs 4.0 International License (CC BY-NC-ND 4.0), which permits the noncommercial replication and distribution of the article with the strict proviso that no changes or edits are made and the original work is properly cited (including links to both the formal publication through the relevant DOI and the license). See: https://creativecommons.org/licenses/by-nc-nd/4.0/.

\section{References}

1. Bugter O, van de Ven SEM, Hardillo JA, et al. Early detection of esophageal second primary tumors using
Lugol chromoendoscopy in patients with head and neck cancer: A systematic review and meta-analysis. Head Neck 2019;41:1122-30.

2. Onochi K, Shiga H, Takahashi S, et al. Risk Factors Linking Esophageal Squamous Cell Carcinoma with Head and Neck Cancer or Gastric Cancer. J Clin Gastroenterol 2019;53:e164-70.

3. Kilciksiz S, Gokce T, Baloglu A, et al. Characteristics of synchronous- and metachronous-type multiple primary neoplasms: a study of hospital-based cancer registry in Turkey. Clin Genitourin Cancer 2007;5:438-45.

4. Matsui T, Okada T, Kawada K, et al. Detection of Second Primary Malignancies of the Esophagus and Hypophraynx in Oral Squamous Cell Carcinoma Patients. Laryngoscope Investig Otolaryngol 2018;3:263-7.

5. Wang YK, Chuang YS, Wu TS, et al. Endoscopic screening for synchronous esophageal neoplasia among patients with incident head and neck cancer: Prevalence, risk factors, and outcomes. Int J Cancer 2017;141:1987-96.

6. Chung CS, Lo WC, Wen MH, et al. Long Term Outcome of Routine Image-enhanced Endoscopy in Newly Diagnosed Head and Neck Cancer: a Prospective Study of 145 Patients. Sci Rep 2016;6:29573.

7. Huang YC, Lee YC, Tseng PH, et al. Regular screening of esophageal cancer for 248 newly diagnosed hypopharyngeal squamous cell carcinoma by unsedated transnasal esophagogastroduodenoscopy. Oral Oncol 2016;55:55-60.

8. Atienza JAS, Dasanu CA. Incidence of second primary malignancies in patients with treated head and neck cancer: a comprehensive review of literature. Curr Med Res Opin 2012;28:1899-909.

9. Hamada Y, Mizuno T, Tanaka K, et al. Esophageal squamous cell neoplasia is an independent negative prognostic factor for head and neck cancer patients. Int J Clin Oncol 2018;23:243-8.

10. Morita M, Egashira A, Nakaji YU, et al. Treatment of Squamous Cell Carcinoma of the Esophagus Synchronously Associated with Head and Neck Cancer. In Vivo 2017;31:909-16.

11. Ko HH, Cheng SL, Lee JJ, et al. Factors influencing the incidence and prognosis of second primary tumors in patients with oral squamous cell carcinoma. Head Neck 2016;38:1459-66.

12. Gong EJ, Kim DH, Ahn JY, et al. Routine endoscopic screening for synchronous esophageal neoplasm in patients with head and neck squamous cell carcinoma: a prospective study. Dis Esophagus 2016;29:752-9. 
13. Blanchard D, Barry B, De Raucourt D, et al. Guidelines update: posttreatment follow-up of adult head and neck squamous cell carcinoma: screening for metastasis and metachronous esophageal and bronchial locations. Eur Ann Otorhinolaryngol Head Neck Dis 2015;132:217-21.

14. Lim H, Kim DH, Jung HY, et al. Clinical significance of early detection of esophageal cancer in patients with head and neck cancer. Gut Liver 2015;9:159-65.

15. Kim DH, Gong EJ, Jung HY, et al. Clinical significance of intensive endoscopic screening for synchronous esophageal neoplasm in patients with head and neck squamous cell carcinoma. Scand J Gastroenterol 2014;49:1486-92.

16. Wang WL, Wang CP, Wang HP, et al. The benefit of pretreatment esophageal screening with image-enhanced endoscopy on the survival of patients with hypopharyngeal cancer. Oral Oncol 2013;49:808-13.

17. Su YY, Chen WC, Chuang HC, et al. Effect of routine esophageal screening in patients with head and neck cancer. JAMA Otolaryngol Head Neck Surg 2013;139:350-4.

18. Lee CT, Chang CY, Lee YC, et al. Narrow-band imaging with magnifying endoscopy for the screening of esophageal cancer in patients with primary head and neck cancers. Endoscopy 2010;42:613-9.

19. Kim JS, Kim BW. Esophageal cancer and head and neck cancer: the earlier, the better. Gut Liver 2015;9:131-2.

20. Bradley PJ, Bradley PT. Searching for metachronous tumours in patients with head and neck cancer: the ideal protocol! Curr Opin Otolaryngol Head Neck Surg 2010;18:124-33.

21. MB Amin, SB Edge, FL Greene, et al. AJCC Cancer Staging Manual. 8th edition. New York: Springer, 2017.

22. Curtius K, Wright NA, Graham TA. An evolutionary perspective on field cancerization. Nat Rev Cancer 2018;18:19-32.

23. Baba Y, Ishimoto T, Kurashige J, et al. Epigenetic field cancerization in gastrointestinal cancers. Cancer Lett

Cite this article as: Wang L, Pang W, Zhou K, Li L, Wang F, Cao W, Meng X. Characteristics of esophageal cancer in patients with head and neck squamous cell carcinoma. Transl Cancer Res 2021;10(5):1954-1961. doi: 10.21037/tcr-20-2880
2016;375:360-6.

24. Angadi PV, Savitha JK, Rao SS, et al. Oral field cancerization: current evidence and future perspectives. Oral Maxillofac Surg 2012;16:171-80.

25. Cancer Genome Atlas Research Network; Analysis Working Group: Asan University; BC Cancer Agency, et al. Integrated genomic characterization of oesophageal carcinoma. Nature 2017;541:169-75.

26. Iwatsubo T, Ishihara R, Morishima $T$, et al. Impact of age at diagnosis of head and neck cancer on incidence of metachronous cancer. BMC Cancer 2019;19:3.

27. Liu Y, Chen H, Sun Z, et al. Molecular mechanisms of ethanol-associated oro-esophageal squamous cell carcinoma. Cancer Lett 2015;361:164-73.

28. Wang YK, Chen WC, Lai YH, et al. Influence of Tea Consumption on the Development of Second Esophageal Neoplasm in Patients with Head and Neck Cancer. Cancers (Basel) 2019;11:387.

29. Chung CS, Liao LJ, Lo WC, et al. Risk factors for second primary neoplasia of esophagus in newly diagnosed head and neck cancer patients: A case-control study. BMC Gastroenterol 2013;13:154.

30. Ikawa H, Tonogi M, Yamane GY, et al. Upper Gastrointestinal Tract Cancers as Double-Cancers in Elderly Patients with Oral Squamous Cell Carcinoma. Bull Tokyo Dent Coll 2012;53:9-16.

31. Bugter O, van Iwaarden DLP, Dronkers EAC, et al. Survival of patients with head and neck cancer with metachronous multiple primary tumors is surprisingly favorable. Head Neck 2019;41:1648-55.

32. Joo YH, Cho JK, Koo BS, et al. Guidelines for the Surgical Management of Oral Cancer: Korean Society of ThyroidHead and Neck Surgery. Clin Exp Otorhinolaryngol 2019;12:107-44.

33. Herranz González-Botas J, Varela Vazquez P, Vazquez Barro C. Second primary tumours in head and neck cancer. Acta Otorrinolaringol Esp 2016;67:123-9. 